\title{
Geometric Analysis of Shapes in Ion Mobility-Mass Spectrometry
}

Jean R. N. Haler ${ }^{1,2, r^{*}}$, Eric Béchet ${ }^{3, \dagger}$, Christopher Kune ${ }^{1}$, Johann Far ${ }^{1}$, Edwin De Pauw ${ }^{1}$ ${ }^{1}$ Mass Spectrometry Laboratory, University of Liège, MolSys Research unit, Quartier Agora, Allée du Six Aout 11, B-4000 Liège, Belgium

${ }^{2}$ Luxembourg Institute of Science and Technology - LIST, Materials Research \& Technology MRT Department, L-4422 Belvaux, Luxembourg

${ }^{3}$ Aerospace \& Mechanical Engineering Department, Computer-aided Geometric Design, University of Liège, B-4000 Liège, Belgium

$\dagger J . R . N$. Haler and E. Béchet equally contributed to this work

*Corresponding author email address: jean.haler@uliege.be

\section{Supporting Information 1}




\section{Materials and Methods}

\section{Polymers.}

Poly(ethylene oxide) PEO $\left(\mathrm{CH}_{3} \mathrm{O}-\mathrm{PEO}-\mathrm{H}\right)$ polymers (Figure $\left.\mathrm{S} 1\right)$ were bought from SigmaAldrich (St. Louis, USA). Average molar masses of the samples were 750, 2000 and 5000 g/mol. Poly(2-ethoxy-1,3,2-dioxaphospholane 2-oxide) PEtP (or poly(ethoxyphosphate), Figure S1) was synthesized according to literature. ${ }^{1,2}$
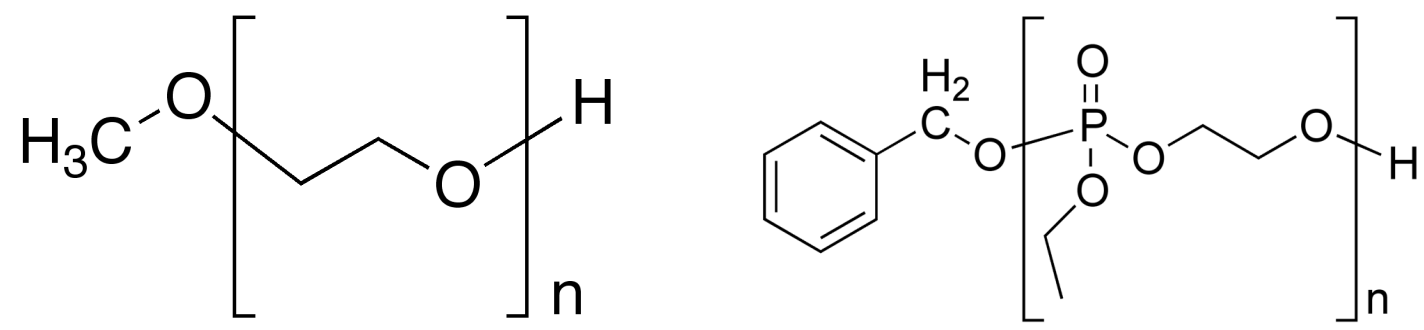

Figure S1: Poly(ethylene oxide) PEO (left) and poly(2-ethoxy-1,3,2-dioxaphospholane 2oxide) or poly(ethoxyphosphate) PEtP polymer (right)

\section{Polymer Ion Mobility-Mass Spectrometry.}

The polymer samples were dissolved in pure methanol (Biosolve) spiked with sodium cations $(\mathrm{NaCl}$ salt $)$ to obtain concentrations of around $10^{-6}$ to $5 \times 10^{-6} \mathrm{M}$ in polymer-sodium complexes.

The samples were infused into a traveling wave ion mobility mass spectrometer (Synapt G2 HDMS from Waters, UK) equipped with an electrospray ionization source (ESI) used in positive mode. The capillary voltage was set to $3 \mathrm{kV}$, the sampling cone voltage was set to $40 \mathrm{~V}$ and the extraction cone was $4 \mathrm{~V}$. The desolvation gas flow was $500 \mathrm{~L} / \mathrm{h}$. The source and desolvation temperatures were set to $100{ }^{\circ} \mathrm{C}$ and $200{ }^{\circ} \mathrm{C}$, respectively. The trap collision energy and the transfer collision energy (CE) were set to $4 \mathrm{~V}$ and $2 \mathrm{~V}$, 
respectively. The trap bias was $45 \mathrm{~V}$. The IMS wave height was $40 \mathrm{~V}$ and the wave speed was set to $1200 \mathrm{~m} / \mathrm{s}$. The trap argon gas flow was $2 \mathrm{~mL} / \mathrm{min}$, the helium gas flow was 180 $\mathrm{mL} / \mathrm{min}$, and the nitrogen pressure in the IM cell was set to 2.6 mbar.

In order to convert drift times into CCS values, a CCS calibration procedure was followed ${ }^{3}$, using reference values obtained in He to calibrate $\mathrm{N}_{2}$ T-Wave measurements ${ }^{4-9}$. IM-MS data were interpreted using Waters' MassLynx 4.1 software. The arrival time distribution (ATD) peaks were fitted using PeakFit v.4.11 to extract accurate drift times. Data processing was performed using Excel 2011 and IgorPro 6.37.

\section{$\left[\mathrm{FeCl}_{3} \mathrm{H}\left(\mathrm{CH}_{2}\right)_{n} \mathrm{CO}_{2}\right]^{-}$clusters Ion Mobility-Mass Spectrometry.}

Ferric(III) chloride, $\mathrm{FeCl}_{3}$, were bought from Sigma-Aldrich (St. Louis, USA). Carboxylic acid used in this study (i.e., acetic acid, propanoic acid, butanoic acid, hexanoic acid, octanoic acid and dodecanoic acid were kindly provided by Professor L. Delaude and Professor A. Demonceau from the Laboratory of Catalysis (University of Liege). $\mathrm{FeCl}_{3}$ were solubilized in methanol from Sigma-Aldrich (St. Louis, USA). Acetic acid, propanoic acid, butanoic acid were solubilized in water while hexanoic acid, octanoic acid and dodecanoic acid were solubilized in methanol. Solution of $\mathrm{FeCl}_{3}$ and one carboxylic acid (one solution for each carboxylic acid) were prepared in 50\% methanol solution to reach a final concentration of 5 and $50 \mu \mathrm{M}$, respectively.

The clusters were infused in the same ion mobility mass spectrometer as the polymer samples. The capillary voltage was set to $-2.2 \mathrm{kV}$, the sampling cone voltage was set to $-30 \mathrm{~V}$ and the extraction cone was $-3 \mathrm{~V}$. The desolvation gas flow was $500 \mathrm{~L} / \mathrm{h}$. The source

and desolvation temperatures were set to $150^{\circ} \mathrm{C}$ and $200^{\circ} \mathrm{C}$, respectively. The trap bias 
was $35 \mathrm{~V}$. The IMS wave height was $40 \mathrm{~V}$ and the wave speed was set to $2100 \mathrm{~m} / \mathrm{s}$. The nitrogen pressure in the IMS cell was set to 2.58 mbar. The helium window (before TWIMS cell) was filled with helium at a pressure of $5.4 \times 10^{-1}$ mbar and the TRAP and TRANSFER cells, placed before and after the TWIMS cell respectively, were filled with argon at a pressure of $2.5 \times 10^{-1}$ mbar. The TWIMS instrument was calibrated in negative mode using polyalanine anions as calibrating substances. The ${ }^{\mathrm{TW}}{ }^{1 \mathrm{ry}} \mathrm{CCS}_{\mathrm{N} 2}$ values were reported by Forsythe and coworkers ${ }^{10}$ (in $\mathrm{N}_{2}$ ), and were considered for this calibration to obtain the $\mathrm{TW}$, ${ }^{2 r y} \mathrm{CCS}_{\mathrm{N} 2}$ values (secondary calibration from TWIMS values ${ }^{11}$ ) of [FeCl3H(CH2)nCO2]clusters ions.

\section{MoShade mathematical background.}

In brief, MoShade calculates the projected area of the shape by sampling its orientation based on the angle $\theta$ and the rotation around the axis $\phi$ (Figure 1). Eq. S1 yields the average projected area $\bar{S}_{p} \cdot \bar{S}_{p}$ is the integral of the cross-section (projected area) for each orientation $(\theta, \phi)$, normalized (or weighted) by the probability to find the shape in one orientation or the other. This probability depends on the solid angle covered by an infinitesimal area on the unit sphere. This area is $2 \pi \cos \theta d \theta$. The projected area is therefore integrated and divided by the integral of the probability density. Because of symmetries, we can restrict the computation of the average $\bar{S}_{p}$ to $1 / 4$ of the total solid angle (sphere) covered by the variables $(\theta, \phi)$. Eq. S1 can be rewritten as Eq. S2 because of symmetries, mainly because a shadow 'from below' has the same area as a shadow 'from above' for any given shape. 


$$
\begin{aligned}
& \bar{S}_{p}=\frac{\int_{-\pi-\pi / 2}^{\pi} \int_{p}^{\pi / 2} S_{p}(\theta, \phi) 2 \pi \cos \theta d \theta d \phi}{\int_{-\pi}^{\pi} \int_{-\pi / 2}^{\pi / 2} 2 \pi \cos \theta d \theta d \phi}=\frac{1}{4 \pi} \int_{-\pi}^{\pi} \int_{-\pi / 2}^{\pi / 2} S_{p}(\theta, \phi) \cos \theta d \theta d \phi \\
& \bar{S}_{p}=\frac{1}{\pi} \int_{0}^{\pi} \int_{0}^{\pi / 2} S_{p}(\theta, \phi) \cos \theta d \theta d \phi
\end{aligned}
$$

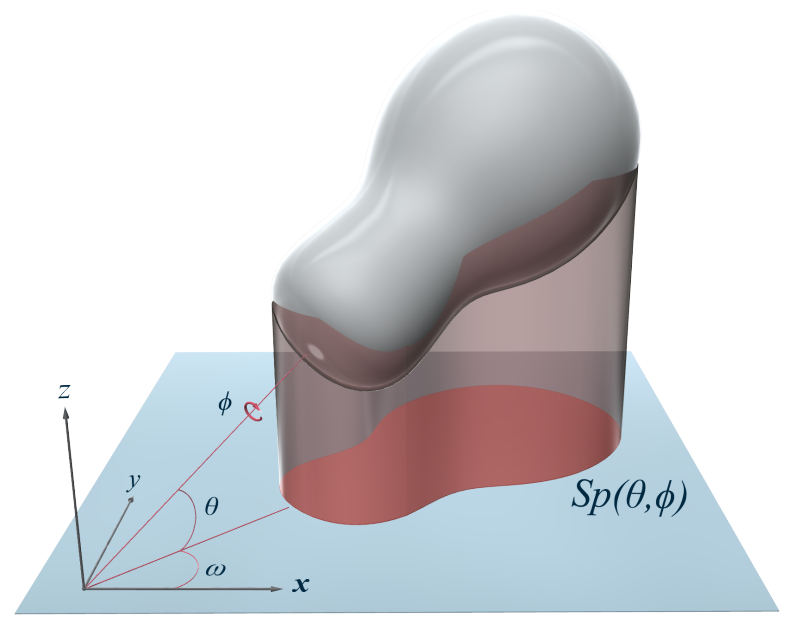

Figure S2: Illustration of a shape projection (in red below the white/gray shape) as performed by MoShade. The angles $\theta$ and rotation axis $\phi$ describe the shape's orientation and are sampled during the projection calculations. $\omega$ is the angle in the $(x, y)$ plane. The shape input is given as a mesh and the projected area is calculated as the sum of the projections of each of the mesh's triangles (see text).

If one wishes to use numerical integration instead of trying to integrate analytically, one may use Eq. S3 as simple quadrature of Eq. S2, with a uniform repartition of the $N$ samples on $\theta_{i}, \phi_{i} \in\left[0 \ldots \frac{\pi}{2}\right] \times[0 \ldots \pi]$. The $n$ samples parameterizing a MoShade calculation hence lead to $N=(2 n)^{2}$. 
$\overline{S_{p}}=\frac{1}{\pi} \int_{0}^{\pi} \int_{0}^{\pi / 2} S_{p}(\theta, \phi) \cdot \cos \theta d \theta d \phi \approx \frac{1}{\pi} \sum_{i} \quad \frac{\pi^{2}}{2 N} S_{p}\left(\theta_{i}, \phi_{i}\right) \cdot \cos \theta_{i}$

To evaluate $S_{p}\left(\theta_{i}, \phi_{i}\right)$, the surface of the shape must be decomposed into a mesh of simple geometrical elements (e.g. triangles or quads). Therefore MoShade uses mesh input files, where the shape is subdivided into small flat triangles (.stl files). The difficulty here is to compute the merged projected area for each triangle 'at once', i.e. by taking care of the shadowing of some triangles by others. MoShade therefore eliminates triangles that are not oriented adequately (see Supporting Information 2), and computes the intersection between the projection of each triangle and the current projected area for a given shape orientation $\left(\theta_{i}, \phi_{i}\right)$, and adds only the 'not already covered' area, if it exists.

However, directly using the quadrature in Eq. S3 yields numerical issues. Indeed, the result is inaccurate when integrating a constant cross-section coming from a spherical shape for instance. This bad behavior originates from the term in $\cos \theta$. One could expect an exact result even with only one sample point in this case, as for any reasonably good quadrature such as Gaussian integration. To reach this goal (i.e. an exact result), the integration is optimized using a change of variables (see Supporting Information 2 for more details). The term $\cos \theta$ is cancelled in the integrand so that $\cos \theta d \theta$ is seen as constant. By changing $\theta=\sin ^{-1} \psi$, and by substituting the boundaries of the integral in Eq. S2 to their sinus, one obtains Eq. S4, which constitutes the optimized integration used by MoShade. Once again, the $n$ samples parameterizing a MoShade calculation lead to $N=(2 n)^{2}$.

$$
\bar{S}_{p}=\frac{1}{\pi} \int_{0}^{\pi} \int_{0}^{1} S_{p}\left(\sin ^{-1} \psi, \phi\right) d \psi d \phi \approx \frac{1}{N \pi} \sum_{i} S_{p}\left(\sin ^{-1} \psi, \phi_{i}\right)
$$

It should be noted that the change of variable works because the bounds of the integral are such that the application $\theta=\sin ^{-1} \psi$ is monotonous in the interval. 


\section{Results and Discussions}
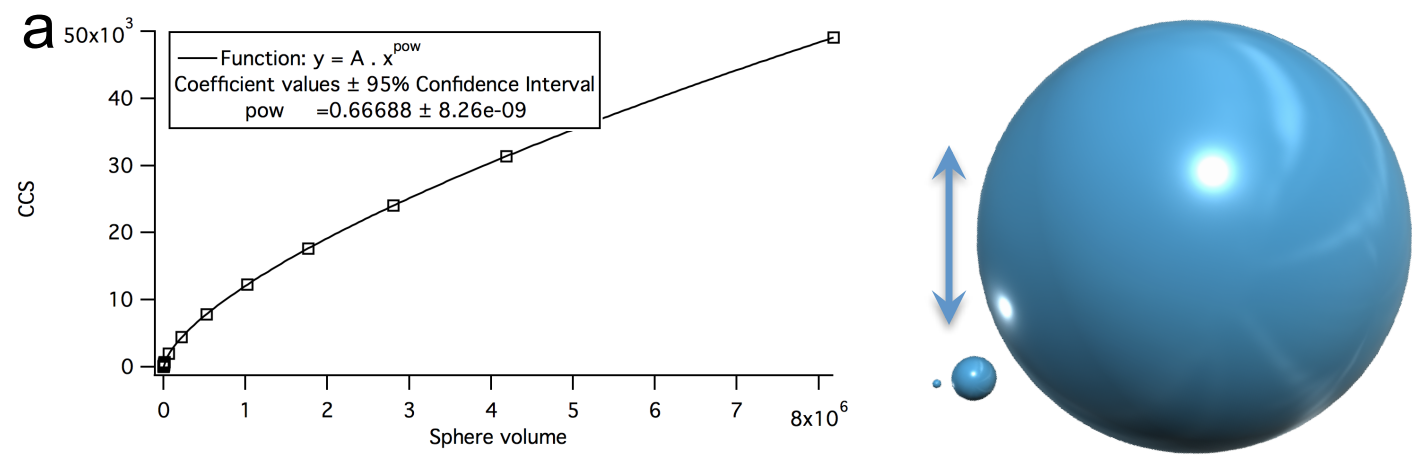

b
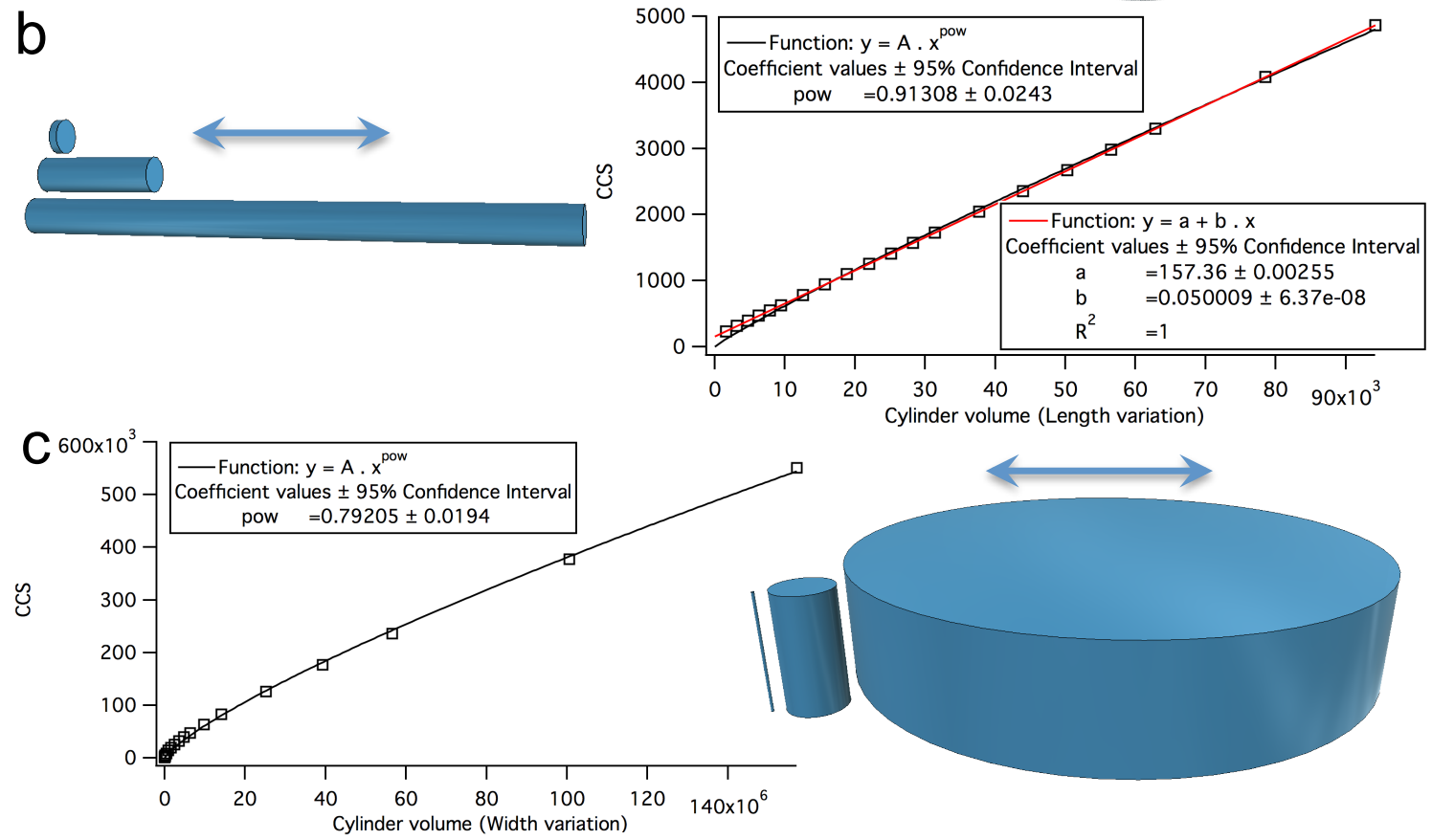

d
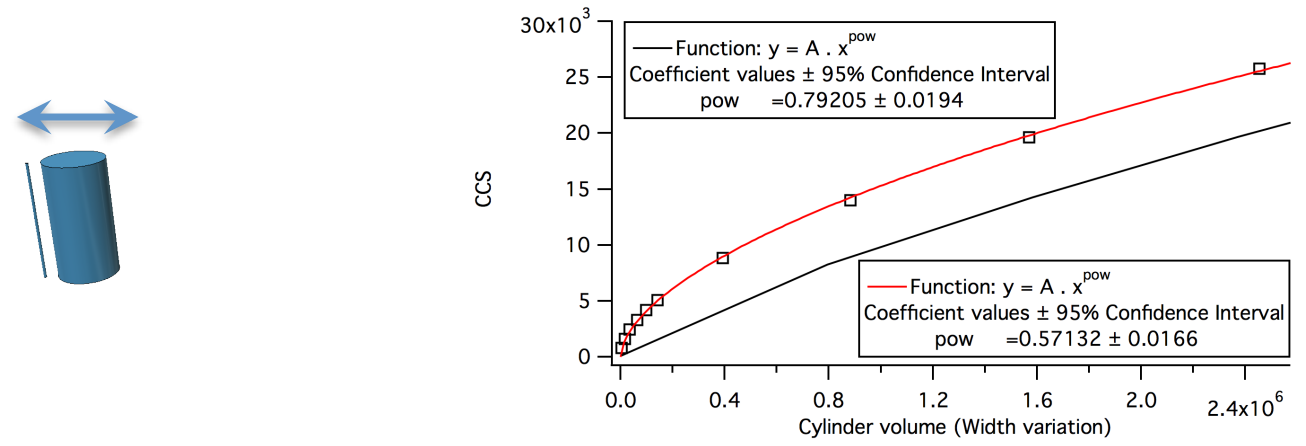

Figure S3: Plots of the Collision Cross-Section (CCS) calculated using MoShade as a function of the shape volume. The fit functions of the CCS evolutions (arbitrary units) are either power functions or linear functions. The structures accompanying each plot represent 
the smallest considered structure, an intermediate size structure (except for S2.d) and the largest structure of each series. The plots depict: S2.a a sphere with a growing diameter; S2.b a cylinder with an increasing cylinder length; S2.c a cylinder with an increasing cylinder diameter and S2.d an extract of S2.c at small CCS an volume values with a more appropriate fit weighted at smaller values (represented in red). Table 1 sums up the shape variations with the minimum and maximum dimensions, as well as the number of samples and the fit parameter values. 

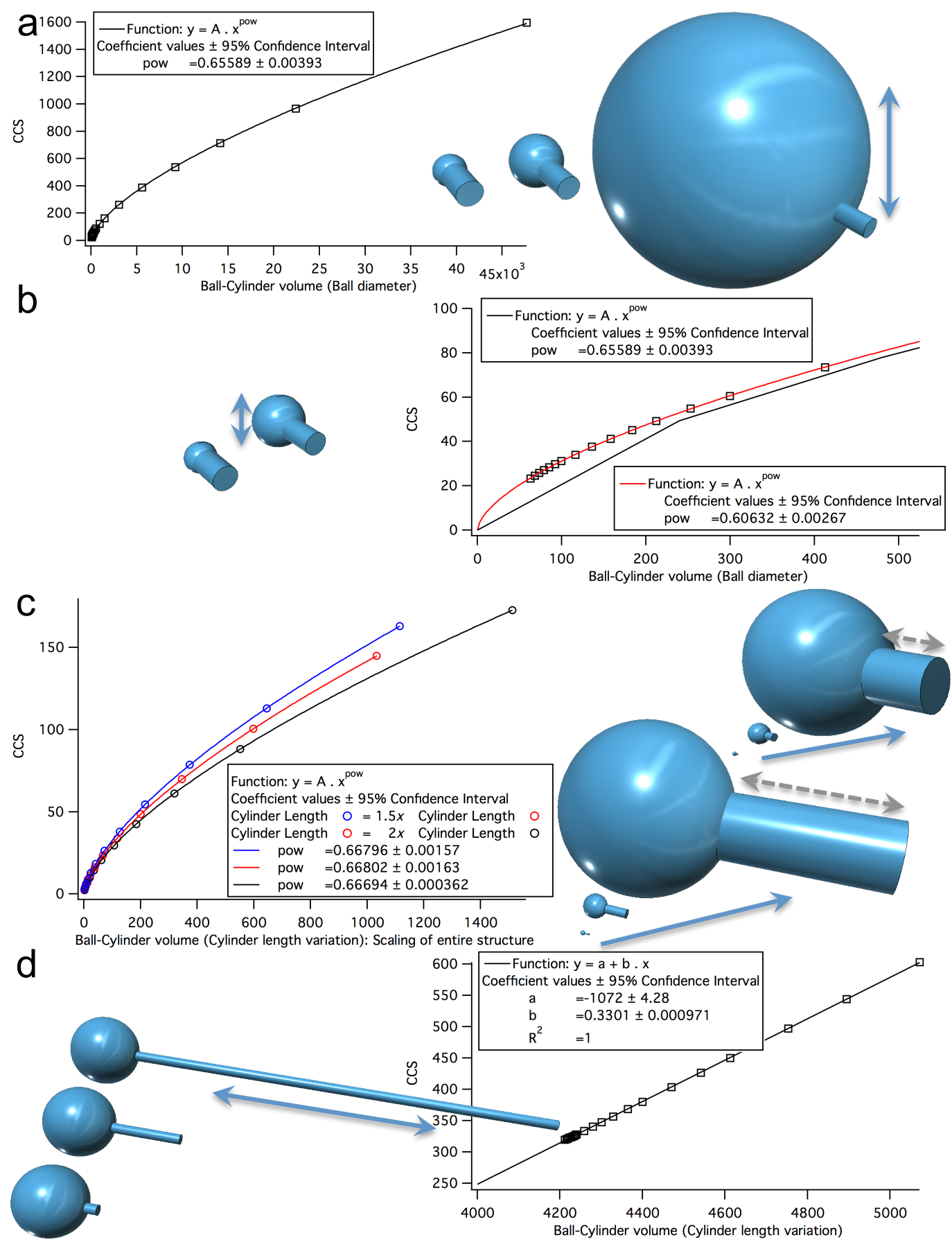

Figure S4: Plots of the Collision Cross-Section (CCS) calculated using MoShade as a function of the shape volume. The fit functions of the CCS evolutions (arbitrary units) are either power functions or linear functions. The structures accompanying each plot represent the smallest considered structure, an intermediate size structure (except for S3.b) and the 
largest structure of each series. The plots depict composite ball-cylinder shapes: S3.a ballcylinder with a growing ball diameter; S3.b an extract of S3.a at small CCS an volume values with a more appropriate fit weighted at smaller values (represented in red); S3.c ball-cylinders with differing cylinder lengths which undergo a uniform isotropic shape increase/scaling; S3.d a ball-cylinder with an increasing cylinder length. Table 1 sums up the shape variations with the minimum and maximum dimensions, as well as the number of samples and the fit parameter values.

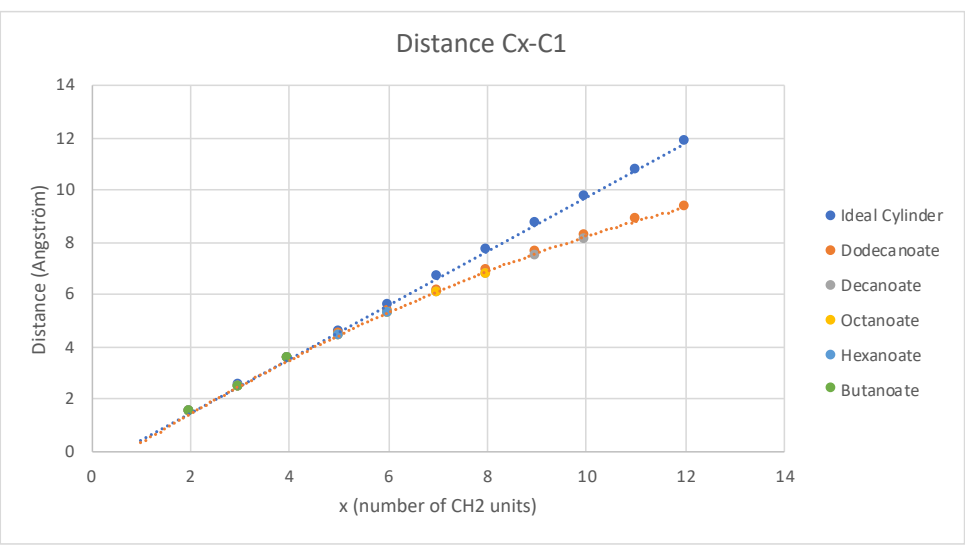

Figure S5: Distance evolutions by $-\mathrm{CH}_{2}$ - unit increments for the case of an ideal cylinder (in blue; linear evolution) and for MD-modeled carboxylate ions (without $\mathrm{FeCl}_{3}$ core; median distance calculated from 4000 structures extracted over 100 nanoseconds at 500 $\mathrm{K})$. The distance is calculated as the difference between an $x^{\text {th }} \mathrm{CH}_{2}$ unit in the chain and the first $\mathrm{C}$ from the carboxylate group " $C_{x}-C_{1}$ ". 
Table S1: Structure pools generated by MM2 and MMFF94 force fields implemented in Chem3D Pro v.11.0 with and without structure optimization. a. $\left[\mathrm{PEO}_{\mathrm{DP}=14}+1 \mathrm{Na}^{+}\right]^{1+}$ and b. $\left[\mathrm{PEO}_{\mathrm{DP}=14}+2 \mathrm{Na}^{+}\right]^{2+}$ structure candidates (represented in 2 different viewing angles). The structures pool is obtained by sampling different simulation temperatures. The last column of the table gives the value of the $S / C C S$ ratio ( $S$ as the geometric surface area) of each structure, as calculated by MoShade (arbitrary units). The bold values represent the structures providing the best agreement with Eq. $9(S=4 . C C S)$. These structures can be correlated to optimized structure geometries. 


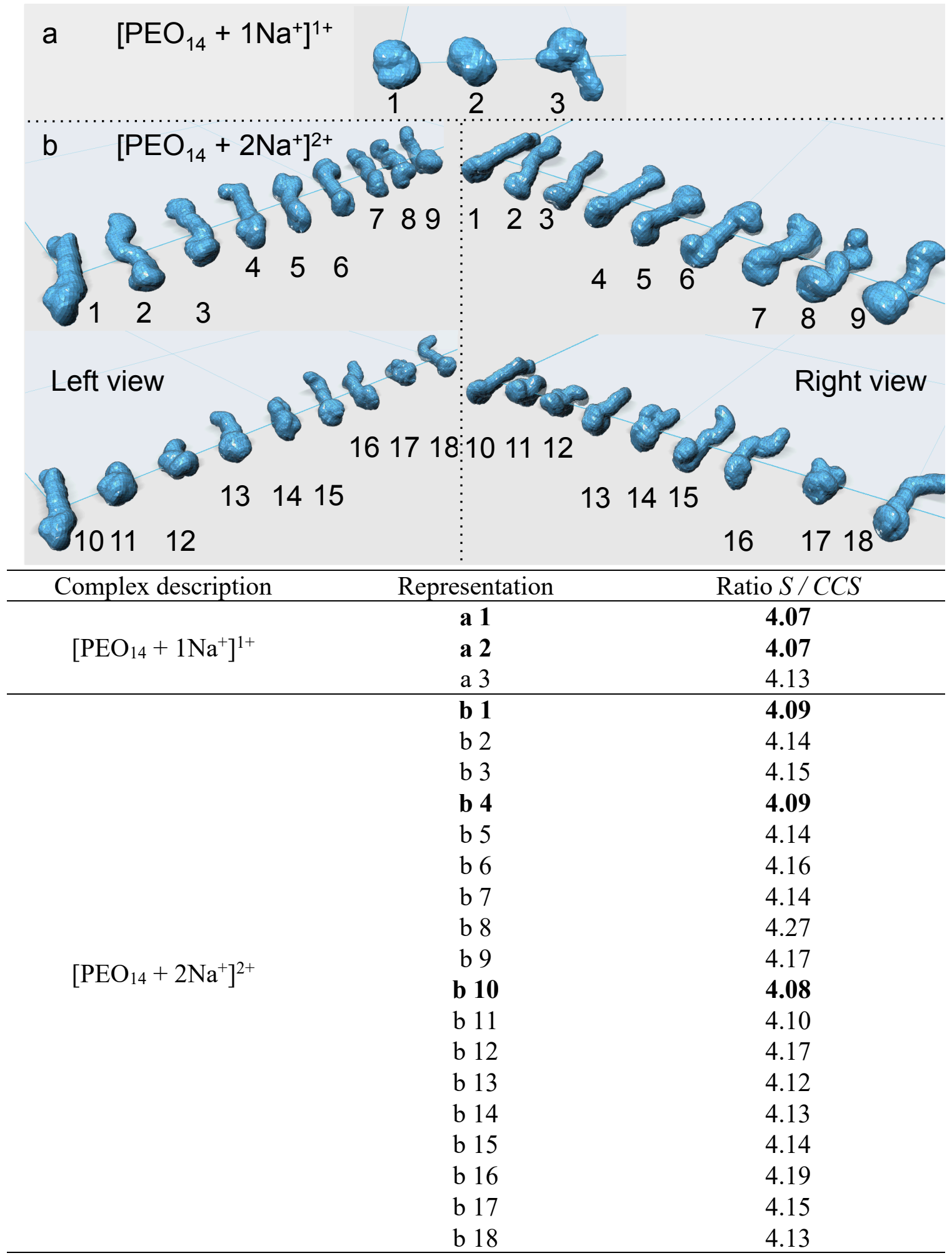


Table S2: Slopes of the linear fits $(b)$ of the CCS plotted as a function of the volume of all the modeled beads on a string shapes (see Figure 4.c in the main article). The dimensions of the bead-string unit are given as descriptors. BD represents the ball diameter, $\mathrm{CD}$ represents the cylinder diameter and CL represents the cylinder length. The ratios leading to the best agreement with the experimental ratio between PEtP and PEO (see main text) are written in bold.

\begin{tabular}{|c|c|c|c|c|c|c|c|c|c|c|c|}
\hline \multicolumn{3}{|c|}{$\begin{array}{c}\text { Description } \\
B D-C D-C L\end{array}$} & $\begin{array}{l}0 \\
1 \\
0 \\
1 \\
\infty\end{array}$ & $\begin{array}{c}\mathcal{1} \\
1 \\
0 \\
1 \\
\infty\end{array}$ & 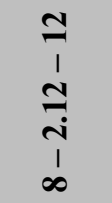 & $\begin{array}{l}m \\
1 \\
m \\
1 \\
\tilde{N}\end{array}$ & $\begin{array}{l}0 \\
1 \\
m \\
1 \\
0 \\
\text { N }\end{array}$ & $\begin{array}{l}1 \\
1 \\
\text { m } \\
1 \\
\text { ᄀิ }\end{array}$ & $\begin{array}{l}m \\
1 \\
0 \\
1 \\
\tilde{N}\end{array}$ & $\begin{array}{l}0 \\
1 \\
0 \\
1 \\
\text { d }\end{array}$ & 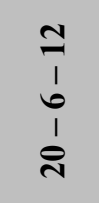 \\
\hline & \multirow{2}{*}{$\begin{array}{c}\text { Slope } \\
\boldsymbol{b} \\
\boldsymbol{b}_{1} \\
\end{array}$} & $b_{2}$ & 0.190 & 0.209 & 0.216 & 0.068 & 0.071 & 0.075 & 0.068 & 0.071 & 0.076 \\
\hline & & $b_{1} / b_{2}$ & & & & & & & & & \\
\hline $8-3-6$ & 0.190 & & 1 & 0.910 & 0.880 & 2.782 & 2.686 & 2.531 & 2.789 & 2.670 & 2.512 \\
\hline $8-3-12$ & 0.209 & & 1.099 & 1 & 0.967 & 3.058 & 2.951 & 2.782 & 3.065 & 2.934 & 2.761 \\
\hline $\begin{array}{c}8-2.12- \\
12\end{array}$ & 0.216 & & 1.136 & 1.034 & 1 & 3.161 & 3.051 & 2.876 & 3.169 & 3.033 & 2.854 \\
\hline $20-3-3$ & 0.068 & & 0.359 & 0.327 & 0.316 & 1 & 0.965 & 0.910 & 1.002 & 0.960 & 0.903 \\
\hline $20-3-6$ & 0.071 & & 0.372 & 0.339 & 0.328 & 1.036 & 1 & 0.942 & 1.038 & 0.994 & 0.935 \\
\hline $20-3-12$ & 0.075 & & 0.395 & 0.360 & 0.348 & 1.099 & 1.061 & 1 & 1.102 & 1.055 & 0.992 \\
\hline $20-6-3$ & 0.068 & & 0.359 & 0.326 & 0.316 & 0.998 & 0.963 & 0.908 & 1 & 0.957 & 0.901 \\
\hline $20-6-6$ & 0.071 & & 0.375 & 0.341 & 0.330 & 1.042 & 1.006 & 0.948 & 1.045 & 1 & 0.941 \\
\hline $20-6-12$ & 0.076 & & 0.398 & 0.362 & 0.350 & 1.108 & 1.069 & 1.008 & 1.110 & 1.063 & 1 \\
\hline
\end{tabular}


Table S3: Composite Ball-Cylinder shapes constituted by 3 balls and 2 cylinders. The shape variation is made up of angle variations as shown in the Figure. The corresponding CCS values, calculated using MoShade, and their percentage variations are shown (calculated taking the shape with a $0^{\circ}$ angle as reference).

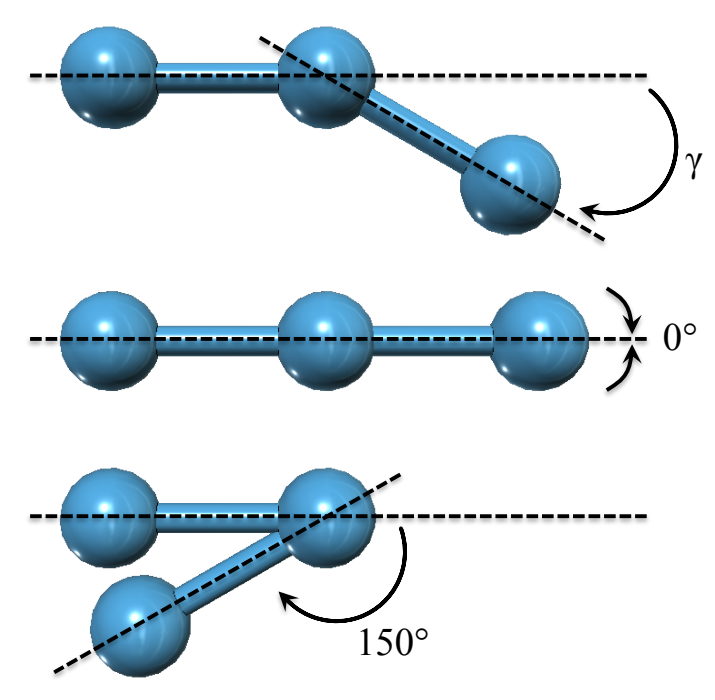

\begin{tabular}{ccc}
\hline Angle $\gamma$ & $\begin{array}{c}\text { CCS } \\
\text { (arbitrary units) }\end{array}$ & $\begin{array}{c}\text { CCS difference in \% } \\
\left.\text { (reference taken at } 0^{\circ}\right)\end{array}$ \\
\hline $0^{\circ}$ & 1092 & 0.0 \\
$15^{\circ}$ & 1095 & -0.2 \\
$30^{\circ}$ & 1092 & 0.1 \\
$45^{\circ}$ & 1087 & 0.5 \\
$60^{\circ}$ & 1089 & 0.3 \\
$75^{\circ}$ & 1087 & 0.5 \\
$90^{\circ}$ & 1086 & 0.5 \\
$105^{\circ}$ & 1081 & 1.0 \\
$120^{\circ}$ & 1073 & 1.8 \\
$135^{\circ}$ & 1061 & 2.9 \\
$150^{\circ}$ & 1031 & 5.7 \\
\hline
\end{tabular}




\section{References}

(1) Clément, B.; Grignard, B.; Koole, L.; Jérôme, C.; Lecomte, P. Metal-Free Strategies for the Synthesis of Functional and Well- De Fi Ned Polyphosphoesters. Macromolecules 2012, 45, 4476-4486. https://doi.org/10.1021/ma3004339.

(2) Haler, J. R. N.; Morsa, D.; Lecomte, P.; Jérôme, C.; Far, J.; De Pauw, E. Predicting Ion Mobility-Mass Spectrometry Trends of Polymers Using the Concept of Apparent Densities. Methods 2018. https://doi.org/10.1016/j.ymeth.2018.03.010.

(3) Ruotolo, B. T.; Benesch, J. L. P. P.; Sandercock, A. M.; Hyung, S.-J. J.; Robinson, C. V. Ion Mobility-Mass Spectrometry Analysis of Large Protein Complexes. Nat. Protoc. 2008, 3 (7), 1139-1152. https://doi.org/10.1038/nprot.2008.78.

(4) Counterman, A. E.; Valentine, S. J.; Srebalus, C. A.; Henderson, S. C.; Hoaglund, C. S.; Clemmer, D. E. High-Order Structure and Dissociation of Gaseous Peptide Aggregates That Are Hidden in Mass Spectra. J. Am. Soc. Mass Spectrom. 1998, 9 (8), 743-759. https://doi.org/10.1016/S1044-0305(98)00052-X.

(5) Bush, M. F.; Campuzano, I. D. G. G.; Robinson, C. V. Ion Mobility Mass Spectrometry of Peptide Ions: Effects of Drift Gas and Calibration Strategies. Anal. Chem. 2012, 84 (16), 7124-7130. https://doi.org/10.1021/ac3014498.

(6) Valentine, S. J.; Counterman, A. E.; Clemmer, D. E. Conformer-Dependent ProtonTransfer Reactions of Ubiquitin Ions. J. Am. Soc. Mass Spectrom. 1997, 8 (9), 954961. https://doi.org/10.1016/S1044-0305(97)00085-8.

(7) Shelimov, K. B.; Jarrold, M. F. Vacuum: An Activation Barrier for Gas-Phase Protein Folding. J. Am. Chem. Soc. 1997, 119 (13), 2987-2994.

(8) Chen, Y. L.; Collings, B. A.; Douglas, D. J. Collision Cross Sections of Myoglobin 
and Cytochrome c Ions with Ne, Ar, and Kr. J. Am. Soc. Mass Spectrom. 1997, 8 (7), 681-687. https://doi.org/10.1016/S1044-0305(97)00033-0.

(9) Valentine, S. J.; Anderson, J. G.; Ellington, A. D.; Clemmer, D. E. Disulfide-Intact and -Reduced Lysozyme in the Gas Phase: Conformations and Pathways of Folding and Unfolding. J. Phys. Chem. B 1997, 101 (19), 3891-3900. https://doi.org/10.1021/jp970217o.

(10) Forsythe, J. G.; Petrov, A. S.; Walker, C. A.; Allen, S. J.; Pellissier, J. S.; Bush, M. F.; Hud, N. V.; Fernández, F. M. Collision Cross Section Calibrants for Negative Ion Mode Traveling Wave Ion Mobility-Mass Spectrometry. Analyst 2015, 140 (20), 6853-6861. https://doi.org/10.1039/c5an00946d.

(11) Gabelica, V.; Shvartsburg, A. A.; Afonso, C.; Barran, P.; Benesch, J. L. P.; Bleiholder, C.; Bowers, M. T.; Bilbao, A.; Bush, M. F.; Campbell, J. L.; Campuzano, I. D. G.; Causon, T.; Clowers, B. H.; Creaser, C. S.; De Pauw, E.; Far, J.; FernandezLima, F.; Fjeldsted, J. C.; Giles, K.; Groessl, M.; Hogan, C. J.; Hann, S.; Kim, H. I.; Kurulugama, R. T.; May, J. C.; McLean, J. A.; Pagel, K.; Richardson, K.; Ridgeway, M. E.; Rosu, F.; Sobott, F.; Thalassinos, K.; Valentine, S. J.; Wyttenbach, T.; T. Bowers, M.; Bilbao, A.; F. Bush, M.; Campbell, J. L.; Campuzano, I. D. G.; J. Causon, T.; H. Clowers, B.; Creaser, C. S.; De Pauw, E.; Far, J.; Fernandez-Lima, F.; Fjeldsted, J. C.; Giles, K.; Groessl, M.; Wyttenbach, T. Recommendations for Reporting Ion Mobility Mass Spectrometry Measurements. Mass Spectrom. Rev. 2019, 38 (3), 291-320. https://doi.org/10.1002/mas.21585. 